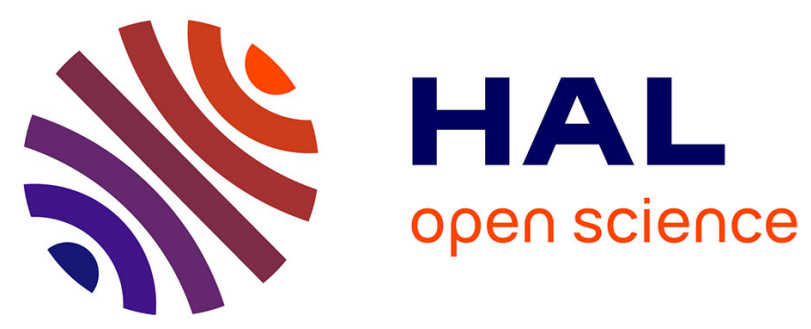

\title{
DECHARGE NON CYLINDRIQUE FOCALISANTE ALIMENTEE PAR UN GENERATEUR D'ENERGIE UTILISANT DE L'EXPLOSIF
}

\author{
J. Bernard, J. Boussinesq, J. Morin, C. Nazet, C. Patou, J. Vedel
}

\section{- To cite this version:}

J. Bernard, J. Boussinesq, J. Morin, C. Nazet, C. Patou, et al.. DECHARGE NON CYLINDRIQUE FOCALISANTE ALIMENTEE PAR UN GENERATEUR D'ENERGIE UTILISANT DE L'EXPLOSIF. Journal de Physique Colloques, 1971, 32 (C5), pp.C5b-90-C5b-92. 10.1051/jphyscol:1971586 . jpa-00214805

\section{HAL Id: jpa-00214805 https://hal.science/jpa-00214805}

Submitted on 1 Jan 1971

HAL is a multi-disciplinary open access archive for the deposit and dissemination of scientific research documents, whether they are published or not. The documents may come from teaching and research institutions in France or abroad, or from public or private research centers.
L'archive ouverte pluridisciplinaire HAL, est destinée au dépôt et à la diffusion de documents scientifiques de niveau recherche, publiés ou non, émanant des établissements d'enseignement et de recherche français ou étrangers, des laboratoires publics ou privés. 
DECHAROE NON CYIINDRIQUE FOCALISANUE ALIMEMTEE PAR UN GENERATEUR D'ENERGIE UTILISANT DE L'EXPLOSIF

J. BERNARD, J. BOUSSINESQ, J. MORIN, C. NAZET, C. PATOU, J. VEDEL

Commlssarlat à l'Energie Atomlque, Centre d'Etudes de Limel I

B.P. $n^{2} 27,94$ - villeneuve-Saint-Georges

Résumé

La décharge Focus alimentée à l'aide d'un générateur utilisant de l'explosif à été expérimentée avec succès. Lors des premières expérlences, le courant de la décharge a été volontairement limité à une valeur inférleure à l MA pour comparer les résultats obtenus au même niveau de courant lorsque la source d'énergle est un banc de condensateurs. Nous envisageons de délivrer à la décharge des courants supérleurs à $2,5 \mathrm{MA}$.

\section{Abstract}

Focus discharges driven by explosive generators have been successfully fired. The discharge current was deliberately kept lower than I MA In the first experiments to compare the results already obtained with capacitor powered generators. Shots with current larger than 2,5 MA are prepared.

Les décharges électriques conduisant à la formation de plasmas denses ont des temps de fonctionnement de l'ordre de la microseconde. Elles sont généralement allmentées par des bancs de condensateurs rapides. Lorsque l'énergle stockée $\mathrm{E}$ devlent de l'ordre de plusleurs mégajoules, ces bancs sont encombrants et onéreux. Une solution de rechange consiste à ut111ser des générateurs à explosif́s développés à IImell et dans d'autres laboratolres étrangers (Sand1a Corporation, Frascat1, Livermore, et en URSS) (I7. Compte tenu de la forte densité d'énergie de l'explosif $(5 \mathrm{MJ} / \mathrm{kg})$ les générateurs sont de falble volume et peu côtteux ; ils sont évidement détruits a chaque tir mais leur flablilté nous a permis d'envisager leur couplage avec l'expérience Focus [27. Divers laboratolres ont falt croftre l'énergie délivrée à la décharge pour atteindre $400 \mathrm{~kJ}$ environ, ce qui correspond à un courant de $1^{\text {t}}$ ordre de $2,5 \mathrm{MA}$. On constate jusqu'alors que le nombre $N$ de neutrons émis est approximativement proportionnel id $\mathrm{E}^{2}$. Le but des études dont nous reportons les premiers résultats est d'attelndre, par étapes, des courants de l'ordre de $10 \mathrm{MA}$. Signalons que les deux premlers laboratolres étrangers cltés ont déjá alımenté une expérience de plasma, en l'occurence un pinch $\theta$, à l'alde de générateurs de conception volsine $\sqrt{3} 7$.

\section{IE GENERATEUR A EXPLOSIF}

Nous nous contenterons de decrire le générateur utilisé pour la première étape du programme

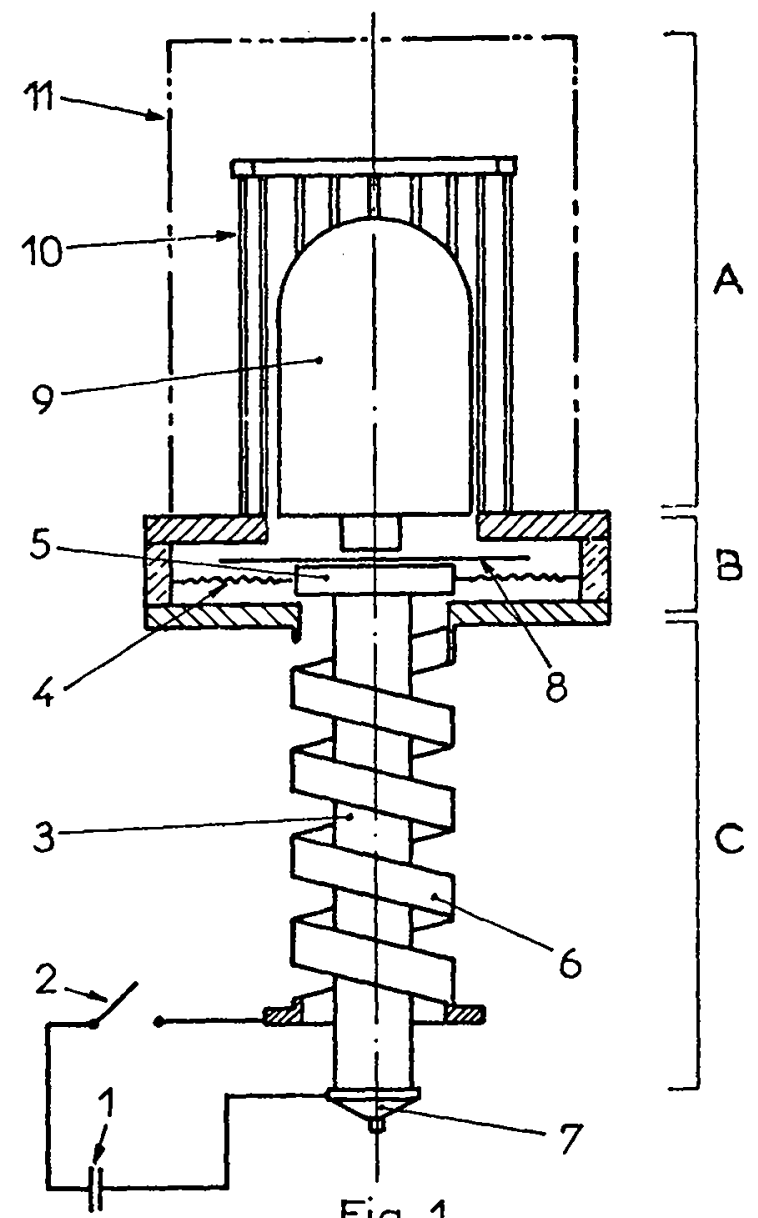

Fig. 1

avec des performances volontalrement limitées. Nous souhations, en effet, dans un premler temps faire fonctionner la décharge avec un courant compris entre 0,5 et 1 MA pour nous permettre de comparer les résultats obtenus, au même niveau de courant lorsque 12 source d'énergle est un barc de condensateurs. 
Le générateur, schématlsé sur $1 a$ partie $C$ de la figure 1, est constitué de deux tubes de culvre coaxlaux. Le tube central (3) de $60 \mathrm{~mm}$ de diamètre est rempll d'explosif $(2,3 \mathrm{~kg}$ pour cette expérience). Le tube extérleur (6) de $150 \mathrm{~mm}$ de diamètre et $55 \mathrm{~cm}$ de long est usiné on forme $d$ 'helice. Le circult électrique est refermé par 32 f1ls de culvre 47 de $0,65 \mathrm{~mm}$ de dlametre régulièrement disposés et dont nous verrons l'ut111 té. I'Inductance du générateur vaut $\mathrm{L}_{\mathrm{O}}=1,35 \mu \mathrm{H}$ et celle des fils $\mathrm{L}_{\mathrm{f}}=0,012 \mu \mathrm{H}$. A l'instant $t=0$ la fermeture de l'Interrupteur (2) permet de dém charger la betterle de condensateurs (1) dans le clrcult électrique décrit qui est aiors parcouru par un courant $I(t)$ reprodult sur la flgure 2 courbe a. Ce courant atteint son maximum $I_{0}=105 \mathrm{kA}$ au temps $t_{0}=60 \mu$. La détonation de l'explosif est amorcée au temps $t_{1}$ à l'extréml té (7) du tube central dont lo relevement contque progresse vers I'extrémité (.5) à la vitesse de détonation de l'explosif. Le contact initial entre le tube relevé et la première spire de l'hélice se prodult au temps $t_{0^{\circ}}$ A cet instant un flux magnetique

$$
\Phi_{0}=\left(L_{0}+I_{f}\right) I_{0}
$$

existe dans le circuit. De $t_{0} t_{2}$ les spires de 1 'hélice sont progressivement court-circuitées et $L\left(t_{2}\right) \approx 0 . S 1 R(t)$ est la résłstance du générateur et $R_{f}(t)$ celle des fils, le courant a pour expresston $[4]$ :

$$
\text { (II) } I(t)=I_{0} \frac{L_{0}+L_{f}}{L(t)+L_{f}} \exp \left[-\int_{t_{0}}^{t} \frac{R(t)+R_{f}(t)}{L(t)} d t\right]
$$

La figure 2 courbe $b$ montre la variation du courant dans le cas où $R_{f}(t)$ ne dépend pas de $t$ $R_{f}=0,36 \cdot 10^{-3} \Omega$. La valour maximale du courant vaut $I\left(t_{2}\right)=3,6 \mathrm{MA}$. Ceci montre que la conservation du flux magnétique, caractérisée par le terme exponentiel de la relation (II) est bien réalisée : cecl est da au fait que le temps de deformation du circult est petit devant le temps de diffusion du champ magnétique. Le temps de fonctionnement total du générateur est de 135 us et on ne peut done pas l'utiliser alnsi pour alimenter une décharge raptde. Un étage intermédiaire, appelé commutateur raplde, est donc nécessalre; nous allons le décrire. Notons que des générateurs du même type, contenant $8 \mathrm{~kg}$ d'explosif convertissent $25 \%$ de l'énergie de l'explosif en énergie électrique et délivrent $25 \mathrm{MA}$ à des inductances de $30 \cdot 10^{-9} \mathrm{H}$.

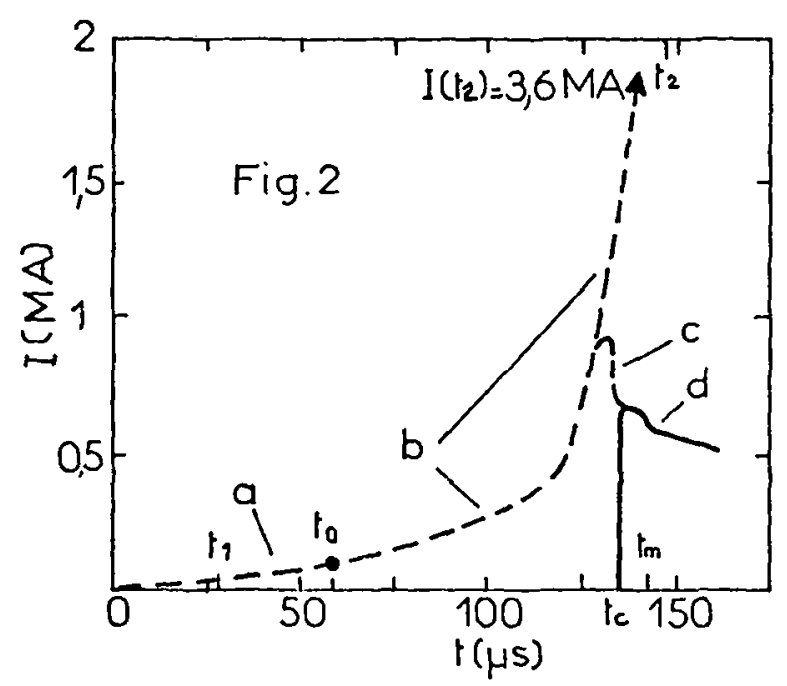

\section{IE COMMUTATEUR RAPIDE}

If est schématisé sur la partie $B$ de la flgure 1. Il comprend un intermpteur a ouverture constitue par les 32 f1ls de culvre (4) et un intermpteur à fermeture à claquage de diélectrique constitué par une feuille de mylar d'épalsseur $26 \mu$ (8). Le nombre et le diemetre des fils, chacun à l'intérieur d'un tube en verre empli de sable, sont calculés pour qu'ils se vaporisent à un temps volsin de $t_{2}$. Leur résistance $R_{f}(t)$ varie donc cette fols dans le temps et le courant du générateur est alors représenté (fig. 2) par la premlère partie de la courbe b puls par la courbe c. Une tension supérleure a $20 \mathrm{kV}$ apparaft aux bornes des f1ls lorsqu'lls se vaporlsent. Par l'intermédialre des blectrodes de la décharge Focus (f1g. 2 - A) cette tension est reportée, sur les faces de la feuille de mylar disposée entre l'électrode intérieure de Focus (9) et l'extrémité du tube central du générateur (5). Le claquage du mylar se produit au temps $t_{c}$ et le courant du générateur est transmis à la décharge. Cette technique nous permet d'atteindre des variations $d u$ courant $\frac{d I}{d t} \approx 10^{12} \mathrm{~A} / \mathrm{s}$. On sa1t, a 2 'heure actuelle comnuter $2,5 \mathrm{MA}$ en $2 \mu \mathrm{s}$. Pour cette prérière expérience, où le courant a été volontairement IImfté, sa variation apparalt sur la figure 2 , courbe d. Elle est très différente de celle produite lorsque la source d'énergle est une batterie de 
condensateurs et se caractérise par une montée très rapide du courant et une décrolssance au contraire plus lente. En changeant la section des fils de cuivre et l'épalsseur de la feullie de mylar on peut modifier la pente du courant. D'autres types $d$ 'interrupteurs à ouverture peuvent être envisagés tel, par exemple, celul à charge creuse isolante 57 dont le jet vient couper un conducteur disposé à la place des fils (4)

\section{EXPERIENCE FOCUS}

Le but des premières expériences est de s'assurer de la possibilité d'utiliser les générateurs en physique des plasmas et plus particulièrement pour fournir le courant à la décharge Focus. Ce point acquis, la façon d'envisager les futures expériences sera très différente. Nous nous sommes tout d'abord préoccupé du fonctionnement correct de la décharge parcourue par un courant ayant l'allure de celut de la figure 2 courbe d. Nous avons donc simuler ce courant, en laboratoire, au niveau de 0,3 MA en remplaçant le générateur à explosif par une batterie de condensateurs, le commutateur rapide découplant cette dernière des électrodes de la décharge. I'expérience ayant été concluante, nous avons couplé la décharge au générateur à explosif selon le schéma de la figure 1. Pour cette série d'expérience les électrodes de la décharge sont détruites à chaque tir. Les dimensions des électrodes et la pression de deutérium sont calculées en utilisant un code numérique [27. Les électrodes sont en cuivre. I'électrode intérieure (9) dont I'extrémité est hémisphérique a un diamètre de $100 \mathrm{~mm}$ et une longueur de $180 \mathrm{~mm}$. Elle est séparée de l'électrode extérieure (10) par un cylindre de verre de $50 \mathrm{~mm}$ de long qui $\mathrm{n}^{\prime}$ est pas représenté sur le schéma 27 . Cette dernlère est formée de colonnettes et son diamètre est de $150 \mathrm{~mm}$. Les électrodes sont enfermées dans une verrerie (11) dans laquelle une pression réslduelle de 0,1 Torr subsiste avant I'introduction du deutérium sous une pression de quelques Torrs. Avant le tir proprement dit un certain nombre de décharges sont effectuées à I'aide d'une batterie de condensateurs rapide pour s'assurer du bon fonctIonnement et dégazer les électrodes. Dès le premier tir nous avons constaté $l^{\prime}$ émission de neutrons et la varlation rapide du courant au temps $t_{m}\left(2\right.$ à $4 \mu$ s après $\left.t_{c}\right)$ ( $f$ is. 2 , courbe d) caractéristique de I'autostriction de la décharge. Pour un courant de 0,7 MA à cet instant le nombre de neutrons émis est de 1 'orare de $1,510^{9}$. Alimentée par une batterie de condensateurs au même niveau de courant la décharge émet en moyenne $4.10^{9}$ neutrons. Nous avons effectué 7 tirs au cours desquels nous avons fait varier la pression de 5 Torrs à 2.5 Torrs. Pour cette dernière pression nous $n$ 'avons pas constaté $d$ 'émission neutronique mals essentiellement pour une raison liée à la physique de I'expérience et non pas au fonctionnement du générateur. Ce dispositif fourni, en effet, un courant qui ne varie pas plus de $10 \% \mathrm{~d}$ 'un tir à I'autre et $11 n^{\prime} y$ a jamals eu de tirs ratés qui leur solt imputable ce qui montre bien la flabilité qu' on peut en attendre.

\section{CONCLUSION}

Au cours de cette année nous envisageons de fourntr 2,5 MA à la décharge. Pour ces expériences les électrodes ne seront plus montées directement en bout du générateur. Le courant commuté sera transporté sur quelques mètres et les électrodes ne seront plus détruites. Après avolr recalé nos résultats sur ceux obtemus à ce niveau dans certains laboratoires nous passerons à l'étape suivante au niveau de 4 à $5 \mathrm{MA}$. Des mesures fines peuvent être envisagées si l'explosion du générateur ne transmet pas de vibrations trop importantes au dispositif expérimental. Une cuve de tir sous vide, comme 11 en existe à I'étranger, fournit une solution à ce problème.

\section{BIBLIOGRAPHIE}

LIJ MORIN (J.), VEDEI (J.) C.R. Acad. Sc. à publier.

LE) PATOU (C.), SIMONRET (A.), WATIEAU (J.P.) Joumal de Phystque, 1968, 29, 973

L37 THOMSON (D.B.) et al., 1967, L.A. 3770, H3

47 CRAWFORD (J.C.), DAMEROW (R.A.), Journal of Applied Physics, 1968, 39, 5224

L5] NAZET (C.), C.R. Acad. Sc., à publier. 